\title{
$\infty \sqrt{1}$ Stroke and \\ In-hospital complications affect short- term and long-term mortality in ICH: a prospective cohort study
}

\author{
Yaqing Zhang (D) , ' Yongjun Wang (D) , ${ }^{1,2}$ Ruijun Ji, ${ }^{1}$ Anxin Wang (D) , ${ }^{2}$ \\ Yilong Wang, ${ }^{1,3}$ Zhonghua Yang, ${ }^{1}$ Liping Liu (D) , ${ }^{1,4}$ Penglian Wang, ${ }^{1}$ \\ Xingquan Zhao, ${ }^{1,2}$ on behalf of the China National Stroke Registry (CNSR) \\ investigators
}

To cite: Zhang Y, Wang Y, Ji R, et al. In-hospital complications affect short-term and long-term mortality in ICH: a prospective cohort study. Stroke \& Vascular Neurology 2021;6: e000386. doi:10.1136/svn-2020-000386

Received 6 April 2020 Revised 10 August 2020 Accepted 30 August 2020 Published Online First 29 October 2020

\section{Check for updates}

(c) Author(s) (or their employer(s)) 2021. Re-use permitted under CC BY-NC. No commercial re-use. See rights and permissions. Published by BMJ.

${ }^{1}$ Department of Neurology, Beijing Tiantan Hospital, Capital Medical University, Beijing,

China

${ }^{2}$ China National Clinical

Research Center for

Neurological Diseases, Beijing,

China

${ }^{3}$ Beijing Key Laboratory of Translational Medicine for Cerebrovascular Disease, Beijing, China

${ }^{4}$ Center of Stroke, Beijing Institute for Brain Disorders, Beijing, China

Correspondence to Dr Penglian Wang; wangpengliantth@163.com

Professor Xingquan Zhao; zxq@vip.163.com

\section{ABSTRACT}

Background Medical complications strongly affected the mortality of patients with stroke. However, only limited research has studied the effect of in-hospital medical complications on the mortality of patients with spontaneous intracerebral haemorrhage (ICH) globally. Using the China National Stroke Registry, the effect was prospectively and systematically investigated in patients with spontaneous ICH during their hospitalisation, at 3, 6 and 12 months after disease onset.

Methods This study collected data on patients over 18 years old with spontaneous ICH from 132 Chinese clinical centres across 32 provinces and four municipalities (Hong Kong included), from September 2007 to August 2008. Data on patient complications, death and other information were acquired through paper-based registry forms. Using multivariable logistic regression, the association of medical complications with stroke outcomes was evaluated.

Results of 3255 patients with spontaneous ICH, 878 (26.97\%) had in-hospital medical complications. Inhospital medical complications were independent risk factors for death during the hospitalisation (adjusted OR $4.41,95 \% \mathrm{Cl} 3.18$ to 6.12 ), at 3 months (adjusted OR 2.18 $95 \% \mathrm{Cl} 1.70$ to 2.80), 6 months (adjusted OR 1.84, 95\% $\mathrm{Cl} 1.45$ to 2.34 ) and 12 months (adjusted OR $1.59,95 \% \mathrm{Cl}$ 1.26 to 2.01) after spontaneous ICH.

Conclusion The results revealed that the short-term and long-term mortality of patients with spontaneous ICH in China was significantly associated with their in-hospital medical complications.

\section{INTRODUCTION}

Spontaneous intracerebral haemorrhage (ICH) is strongly related to the increased rate of mortality and long-term disability worldwide. $^{1-3}$ ICH accounts for $20 \%-30 \%$ of stroke in Asia. ${ }^{45}$ The overall annual incidence of ICH is about 25 per 100000 persons. ${ }^{6}$ Previous studies suggested that approximately $40 \%$ of patients with ICH died within 3 months following a stroke. ${ }^{7-9}$ In addition, previous studies demonstrated that increased mortality and disability of patients who had a stroke were associated with the in-hospital medical complications. ${ }^{10-16}$ This association has been confirmed in the China National Stroke Registry (CNSR) cohort. ${ }^{17-19}$ However, only limited studies investigated the effect of in-hospital medical complications on the mortality of patients with spontaneous ICH worldwide. We therefore prospectively analysed a cohort of 3255 patients with spontaneous ICH in CNSR and investigated whether in-hospital medical complications affected risk of mortality of patients during hospitalisation, at 3, 6 and 12 months after stroke onset.

\section{METHODS}

Data collection and study population

The CNSR is a national, multicentre, prospective observational study designed to investigate patient characteristics, the stroke care system, risk factors and outcomes of patients with acute stroke in China. Additional details regarding study design have been presented previously. ${ }^{20}$ In general, this prospective registry study enrolled patients with acute stroke, including acute ischaemic stroke, ICH, subarachnoid haemorrhage and transient ischaemic attack (TIA). Patients with spontaneous ICH were included in this study. Patients were excluded if they had an ischaemic stroke, subarachnoid haemorrhage, secondary haemorrhage, or TIA and were lost to follow-up. Patient data were collected at baseline, discharge and at 3, 6 and 12 months after the index event. At baseline, a trained investigator interviewed and evaluated each patient and recorded their demographic information, medical history, family history of stroke, baseline National Institutes of Health Stroke Scale (NIHSS) scores, Glasgow Coma Scale (GCS) scores at admission, haematoma volume at admission, haematoma location at admission and other clinical characteristics. The baseline NIHSS score $^{21}$ and GCS score $^{22}$ were used to determine the baseline severity of neurological impairment. Patients' 
complications and other related information were recorded during their hospitalisation. Usually, data for the follow-up were obtained from the patients; in some cases, the information was obtained from their caregiver or surrogates. In this study, all patients with ICH were divided into two groups: those presented with in-hospital medical complications and those without complications.

\section{Definition of medical complications}

In-hospital medical complications were defined as nonneurological complications which occurred during hospitalisation and needed intervention, including pneumonia, decubitus ulcer, gastrointestinal bleeding, deep vein thrombosis and pulmonary embolism, as recorded in the paper-based registry forms. The diagnostic criteria for in-hospital medical complications were explicitly defined to all participating centres. In general, pneumonia was diagnosed with the following criteria: fever, chest symptoms, coarse breathing or crackling sounds in the chest, and was confirmed by a chest X-ray. Deep vein thrombosis was diagnosed if there was: any occurrence swelling in the legs, redness, warmth, and pain, and presence of a deep vein thrombosis by venous Doppler, but cellulitis or infection of the leg had been ruled out. Pulmonary embolism was diagnosed according to the following criteria: chest pain and shortness of breath, sinus tachycardia (heart rate $>100$ beats per minute) as determined by ECG. Urinary tract infections were defined as having: pain or burning during urination, the urge to urinate, cloudy urine and abnormal counts of white and red cells in urinalysis. Gastrointestinal bleeding was defined as having: coffee ground emesis, haematemesis, blood in the nasogastric tube, melena or blood in the rectum. Decubitus ulcer was defined as a skin sore caused by pressure from prolonged lying in bed during hospitalisation.

\section{Ascertainment of outcomes}

In this analysis, the death of a patient was considered a clinical outcome. The outcomes of interest were modified Rankin Scale 6 scores from in-hospital duration to 12-month follow-up. The clinical outcomes of all subjects were assessed by telephone follow-up at 3,6 and 12 months.

\section{Statistical analysis}

Categorical variables were presented as numerical proportions, and continuous variables were presented as means with SD or medians with IQR. Differences between subjects with and without in-hospital complications were tested using a Student's t-test for continuous variables and the $\chi^{2}$ test for categorical variables. To determine the associations between in-hospital medical complications and mortality in patients with spontaneous ICH, univariate logistic regression analyses and multivariate logistic regression analyses were conducted and expressed as unadjusted or adjusted ORs with their corresponding 95\% CIs. Multivariate logistic regression analyses were performed and adjusted for potential covariates including sex, age, NIHSS at baseline and GCS scores, haematoma volume, haematoma location, diabetes mellitus, hypertension, hyperlipidaemia, history of coronary heart disease (CHD), history of TIA, family history of stroke, atrial fibrillation (AF), heavy alcohol intake, current smoking status, dysphagia, anticoagulant treatment, thrombolytic treatment and health insurance type. Missing values were treated as the most categories or categories based on clinical perspectives. For data analysis, SAS V.9.4 statistical software (SAS Institute) was used. A two-tailed probability value $<0.05$ is considered to be statistically significant.

\section{Standard protocol approvals and patient consents}

Written informed consent was obtained from all participants or their surrogates.

\section{Data availability}

Only anonymised data would be shared upon request from the corresponding author.

\section{RESULTS}

This study included 5136 patients with spontaneous ICH. We excluded 1600 individuals with primary ICH (138 patients), ICH of other causes (1462 patients) and those lost to follow-up (281 patients). Finally, 3255 subjects with initial ICH participated until the conclusion of the study (figure 1). The in-hospital prevalence of pneumonia, urinary tract infection, gastrointestinal bleeding, decubitus ulcer, deep vein thrombosis and pulmonary embolism was $17.97 \%, 5.96 \%, 5.50 \%, 0.95 \%, 0.68 \%$ and $0.34 \%$, respectively. Of 3255 patients, $878(26.97 \%)$ had at least one in-hospital medical complication.

The baseline characteristics of enrolled participants are listed in table 1 . The study population was $38.71 \%$ female, and the mean age at onset was $62 \pm 13$ years. Compared with patients without in-hospital medical complications, those with complications were significantly older, had a history of stroke, had higher NIHSS scores but lower GCS scores, had a larger haematoma volume, had higher proportions of being on antihypertension and hypoglycaemic treatment but lower use of lipid-lowering treatment. Additionally, more patients with in-hospital complications had brainstem or lobar haemorrhage. No significant differences were found in the proportion of hypertension, hyperlipidaemia, diabetes mellitus, history of TIA, history of AF, antiplatelet treatment and anticoagulant treatment.

The clinical outcomes of this study during hospitalisation, at 3, 6 and 12 months after spontaneous ICH were also demonstrated in table 1 . The rate of mortality during the hospitalisation, at 3, 6 and 12 months after spontaneous ICH was $9.12 \%$ (297 patients), $19.97 \%$ (650 patients), $22.49 \%$ (732 patients) and $26.11 \%$ (850 patients), respectively. At all time points, the rate of death was notably higher in patients with complications than those without complications.

The univariate logistic regression analyses showed that in-hospital medical complications were significantly 


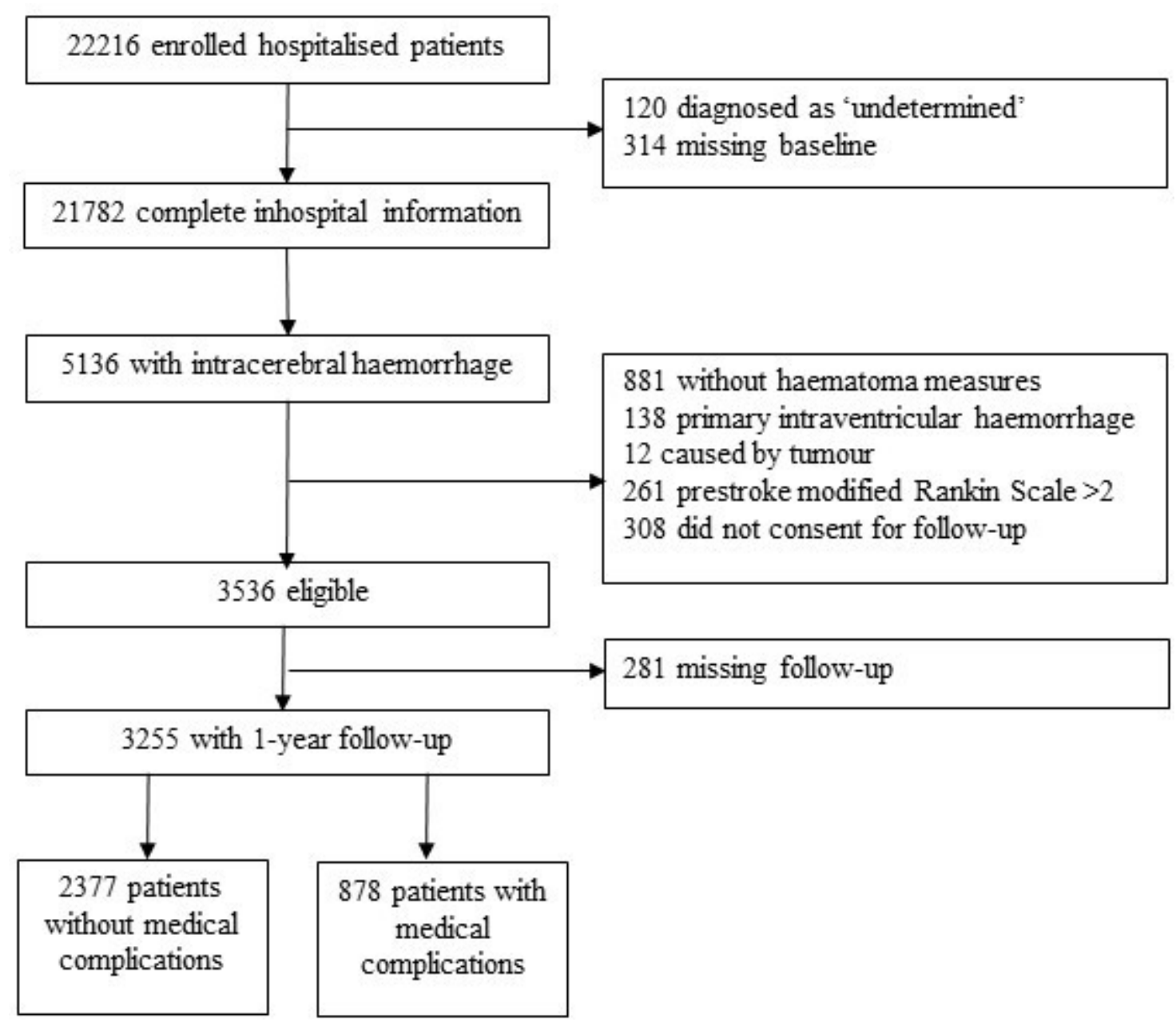

Figure 1 Flow diagram of the study population.

associated with death during hospitalisation, at 3,6 or/ and 12 months after spontaneous ICH (table 2). The multivariate logistic regression analyses showed that pneumonia was associated with a greater risk of death during hospitalisation, at 3 and 6 months after ICH; gastrointestinal bleeding was significantly associated with death at 12 months after ICH. The findings were adjusted for potential covariates including age, sex, baseline NIHSS and GCS scores, haematoma volume, haematoma location, hypertension, diabetes mellitus, hyperlipidaemia, history of TIA, history of CHD, family history of stroke, AF, current smoking status, dysphagia, heavy alcohol intake, anticoagulant treatment, thrombolytic treatment and types of health insurance (table 2).

The unadjusted ORs of death were approximately 5.3fold, 4.1-fold, 3.8-fold and 3.5-fold greater in patients with complication(s) when compared with those without complications during hospitalisation, at 3, 6 and 12 months, respectively. The adjusted ORs of death were approximately 4.4-fold, 2.2-fold, 2.0-fold and 1.9-fold greater in participants with complications when compared with those without complications during hospitalisation, at 3, 6 and 12 months, respectively (table 3 ).

\section{DISCUSSION}

The CNSR is a nationwide prospective stroke registry that enrols high numbers of patients who had a stroke throughout China. In this prospective cohort study, patients with spontaneous ICH from the CNSR were included, and the impact of in-hospital medical complications on the mortality of participants was analysed. The study found that in-hospital medical complications were associated with a higher risk of death in patients with spontaneous ICH in the 12 months following a stroke.

In this study, a total of 878 patients $(26.97 \%)$ had medical complications during their hospitalisation. We found that the frequencies of pneumonia, urinary tract infection, gastrointestinal bleeding, decubitus ulcer, deep vein thrombosis and pulmonary embolism during hospitalisation were $17.97 \%, 5.96 \%, 5.50 \%, 0.95 \%, 0.68 \%$ and $0.34 \%$, respectively. The most common complication was pneumonia, followed by urinary tract infection, then gastrointestinal bleeding. The previously reported incidence of pneumonia varied from $3.5 \%$ to $31.1 \%$ in patients with only spontaneous ICH. ${ }^{923-25}$ Lord et al presented a retrospective cohort of 3550 readmitted patients from non-federal acute care hospitals in California with a 
Table 1 Baseline clinical characteristics and outcomes of the study patients with intracerebral haemorrhage

\begin{tabular}{|c|c|c|c|c|}
\hline Variable & Total $(n=3255)$ & $\begin{array}{l}\text { With any medical } \\
\text { complications }(n=878)\end{array}$ & $\begin{array}{l}\text { Without medical } \\
\text { complications }(n=2377)\end{array}$ & $P$ value \\
\hline Age, mean $\pm S D$ (years) & $62 \pm 13$ & $66 \pm 13$ & $61 \pm 13$ & $<0.0001$ \\
\hline Gender, female, n (\%) & $1260(38.71)$ & $352(40.09)$ & $908(38.20)$ & 0.3254 \\
\hline Hypertension, n (\%) & $2191(67.31)$ & $593(67.54)$ & $1598(67.23)$ & 0.8661 \\
\hline History of stroke, n (\%) & $889(27.31)$ & 272 (30.98) & $617(25.96)$ & 0.0043 \\
\hline History of TIA, n (\%) & $29(0.89)$ & $10(1.14)$ & $19(0.80)$ & 0.3601 \\
\hline History of AF, n (\%) & $54(1.66)$ & $20(2.28$ & $34(1.43)$ & 0.0929 \\
\hline $\begin{array}{l}\text { History of coronary heart disease } \\
(\mathrm{CHD}), \mathrm{n}(\%)\end{array}$ & $204(6.27)$ & $65(7.40)$ & $139(5.85)$ & 0.1042 \\
\hline Antihypertension treatment & 1910 (58.68) & $566(64.46)$ & $1344(56.54)$ & $<0.0001$ \\
\hline Antiplatelet treatment & $31(0.95)$ & $8(0.91)$ & $23(0.97)$ & 0.883 \\
\hline Anticoagulant treatment & $24(0.74)$ & $9(1.03)$ & $15(0.63)$ & 0.2435 \\
\hline Hypoglycaemic treatment & 405 (12.44) & $152(17.31)$ & $253(10.64)$ & $<0.0001$ \\
\hline Lipid-lowering treatment & $275(8.45)$ & $57(6.49)$ & $218(9.17)$ & 0.0147 \\
\hline Current smoking, n (\%) & $825(25.35)$ & 208 (23.69) & $617(25.96)$ & 0.187 \\
\hline Current heavy drinking, $\mathrm{n}(\%)$ & 998 (30.66) & $243(27.68)$ & $755(31.76)$ & 0.0248 \\
\hline Baseline NIHSS, median (IQR) & $10.0(4.0-18.0)$ & $16(9.0-25.0)$ & $8(3.0-14.00)$ & $<0.0001$ \\
\hline GCS at admission, median (IQR) & $14.0(9.0-15.0)$ & $10(6.0-15.0)$ & $15(11-15.0)$ & $<0.0001$ \\
\hline Cerebellum & $184(5.65)$ & $57(6.49)$ & $127(5.34)$ & 0.2077 \\
\hline Death during hospitalisation, $\mathrm{n}(\%)$ & $297(9.12)$ & $184(20.96)$ & $113(4.75)$ & $<0.0001$ \\
\hline Death at 3 months, n (\%) & $650(19.97)$ & $337(38.38)$ & $313(13.17)$ & $<0.0001$ \\
\hline Death at 6 months, $\mathrm{n}(\%)$ & 732 (22.49) & $361(41.12)$ & $371(15.61)$ & $<0.0001$ \\
\hline Death at 12 months, $\mathrm{n}(\%)$ & $850(26.11)$ & $396(45.10)$ & $454(19.10)$ & $<0.0001$ \\
\hline
\end{tabular}

AF, atrial fibrillation; GCS, Glasgow Coma Scale; NIHSS, National Institutes of Health Stroke Scale; TIA, transient ischaemic attack.

primary diagnosis of ICH between 2006 and 2010 and found $124(3.5 \%)$ patients with pneumonia. ${ }^{23}$ Nzwalo $e t$ al reported a retrospective cohort of 549 spontaneous ICH from south Portugal and found $171(31.1 \%)$ patients with in-hospital pneumonia. ${ }^{9}$ Lindner et al prospectively collected a cohort of 229 patients with non-traumatic ICH from the neurocritical care unit (NICU) of a tertiary care hospital in Austria between 2011 and 2016. ${ }^{25}$ They found $64(28 \%)$ patients with pneumonia, which was the most common complication in the study. Morotti et al retrospectively analysed a cohort of 2014 patients with spontaneous ICH from a single academic hospital from 1994 to $2015 .^{24}$ They also confirmed that pneumonia was the most common complication, and the incidence of pneumonia was $18.4 \%$. Our study showed similar results, confirming poor outcome of patients with spontaneous ICH was associated with in-hospital medical complications.

Previous studies mainly focused on 30-day and 3-month risk of death and its association with the medical complications. ${ }^{79} 2326$ In CNSR, the risk of death was increased because of the medical complication(s) during hospitalisation, at 3, 6 and 12 months following spontaneous ICH, expanded the duration of observations. Until now, this study was the largest prospective study on the relationship between in-hospital medical complications and death of patients with spontaneous ICH. 
Table 2 Unadjusted and adjusted ORs for medical complications in relation to the mortality of patients with ICH during hospitalisation, and at 3 months, 6 months and 1 year

\begin{tabular}{|c|c|c|}
\hline Variable & Unadjusted OR (95\% Cl) & Adjusted OR (95\% Cl) \\
\hline \multicolumn{3}{|l|}{ Pneumonia (Yes vs No) } \\
\hline During hospitalisation & 2.41 (1.86 to 3.13$)$ & $2.23(1.59$ to 3.13$)$ \\
\hline At 3 months & 2.59 (2.12 to 3.16$)$ & 1.52 (1.15 to 2.00$)$ \\
\hline At 6 months & $2.56(2.12$ to 3.11$)$ & 1.40 (1.07 to 1.82$)$ \\
\hline At 12 months & $2.42(2.00$ to 2.91$)$ & 1.19 (0.92 to 1.55$)$ \\
\hline \multicolumn{3}{|c|}{ Pulmonary embolism (Yes vs No) } \\
\hline During hospitalisation & 3.77 (0.99 to 14.27$)$ & 2.39 (0.39 to 14.68$)$ \\
\hline At 3 months & $1.51(0.40$ to 5.69$)$ & $0.82(0.12$ to 5.73$)$ \\
\hline At 6 months & 2.89 (0.88 to 9.48$)$ & $1.47(0.27$ to 8.00$)$ \\
\hline At 12 months & $3.41(1.04$ to 11.21$)$ & $2.19(0.43$ to 11.05$)$ \\
\hline \multicolumn{3}{|c|}{ Deep vein thrombosis (Yes vs No) } \\
\hline During hospitalisation & 0.47 (0.06 to 3.52) & 0.84 (0.10 to 6.88$)$ \\
\hline At 3 months & 0.89 (0.30 to 2.64$)$ & $0.92(0.23$ to 3.66$)$ \\
\hline At 6 months & 1.01 (0.37 to 2.76$)$ & 1.09 (0.31 to 3.85$)$ \\
\hline At 12 months & 1.97 (0.84 to 4.63$)$ & 1.64 (0.52 to 5.25$)$ \\
\hline \multicolumn{3}{|c|}{ Urinary tract infection (Yes vs No) } \\
\hline During hospitalisation & $1.30(0.82$ to 2.06$)$ & 1.45 (0.82 to 2.55$)$ \\
\hline At 3 months & $1.23(0.87$ to 1.73$)$ & 1.09 (0.69 to 1.71$)$ \\
\hline At 6 months & $1.25(0.90$ to 1.74$)$ & 1.08 (0.70 to 1.65$)$ \\
\hline At 12 months & $1.32(0.96$ to 1.81$)$ & 1.06 (0.70 to 1.60$)$ \\
\hline \multicolumn{3}{|c|}{ Gastrointestinal bleeding (Yes vs No) } \\
\hline During hospitalisation & 2.31 (1.54 to 3.45$)$ & 1.08 (0.64 to 1.82$)$ \\
\hline At 3 months & 3.38 (2.48 to 4.60$)$ & 1.36 (0.89 to 2.09$)$ \\
\hline At 6 months & 3.56 (2.62 to 4.84$)$ & $1.46(0.96$ to 2.21$)$ \\
\hline At 12 months & 3.74 (2.75 to 5.08$)$ & 1.57 (1.03 to 2.39$)$ \\
\hline \multicolumn{3}{|c|}{ Decubitus ulcers (Yes vs No) } \\
\hline During hospitalisation & 1.93 (0.74 to 5.07$)$ & 1.02 (0.32 to 3.24$)$ \\
\hline At 3 months & 2.93 (1.43 to 6.02$)$ & $1.29(0.52$ to 3.16$)$ \\
\hline At 6 months & 4.26 (2.09 to 8.69$)$ & 1.85 (0.77 to 4.42$)$ \\
\hline At 12 months & 3.98 (1.94 to 8.16$)$ & 1.33 (0.55 to 3.22$)$ \\
\hline
\end{tabular}

Adjusted factors include age, sex, baseline National Institutes of Health Stroke Scale (NIHSS) and Glasgow Coma Scale (GCS) scores, haematoma volume, haematoma location, hypertension, diabetes mellitus, hyperlipidaemia, history of transient ischaemic attack (TIA), history of coronary heart disease (CHD), family history of stroke, atrial fibrillation (AF), current smoking status, dysphagia, heavy alcohol intake, anticoagulant treatment, thrombolytic treatment and types of health insurance. $\mathrm{ICH}$, intracerebral haemorrhage.

Prevention of in-hospital medical complications is key to reduce future death in patients following spontaneous ICH. Such prevention is especially important for patients with vulnerable age, a history of stroke, a higher NIHSS score, a lower GCS score and a larger haematoma volume, who are more likely to have in-hospital medical complications.

Our study had limitations. Only six types of complications were included. In addition, information on the treatment of medical complications was not collected, which may affect the mortality. Finally, all recruited hospitals or centres were from urban areas in China; thus, our results may not be able to represent all levels of hospital care in China.

In conclusion, in patients with spontaneous ICH, pneumonia was the most common in-hospital medical complication. All six in-hospital medical complications were significantly associated with the 12-month mortality in patients with spontaneous ICH. Aggressive treatment and prevention of complications in these patients will prevent future death. Future studies on the mechanisms of complications affecting stroke outcomes are still needed. 
Table 3 Unadjusted and adjusted ORs of having one or more medical complications during hospitalisation for death in patients at various time points following intracerebral haemorrhage

\begin{tabular}{lll}
\hline Outcomes & $\begin{array}{l}\text { Unadjusted OR } \\
\text { (95\% CI) }\end{array}$ & $\begin{array}{l}\text { Adjusted OR (95\% } \\
\text { Cl)* }\end{array}$ \\
\hline $\begin{array}{l}\text { Death during } \\
\text { hospitalisation }\end{array}$ & $5.31(4.14$ to 6.82$)$ & $4.41(3.18$ to 6.12$)$ \\
\hline $\begin{array}{l}\text { Death at } 3 \text { months } \\
\text { Death at } 6 \text { months }\end{array}$ & $4.11(3.43$ to 4.92$)$ & $2.18(1.70$ to 2.80$)$ \\
\hline Death at 12 months & $3.48(2.94$ to 4.12$)$ & $1.59(1.26$ to 2.01$)$ \\
\hline
\end{tabular}

${ }^{*}$ Adjusted factors include age, sex, baseline National Institutes of Health Stroke Scale (NIHSS) and Glasgow Coma Scale (GCS) scores, haematoma volume, haematoma location, hypertension, diabetes mellitus, hyperlipidaemia, history of transient ischaemic attack (TIA), history of coronary heart disease (CHD), family history of stroke, atrial fibrillation (AF), current smoking status, dysphagia, heavy alcohol intake, anticoagulant treatment, thrombolytic treatment and types of health insurance.

Acknowledgements The authors would like to thank the patients who participated in this CNSR study and all investigators, nurses and imaging and laboratory staff in all participating centres. The details of the study design have been published previously.

Contributors Conception and design: YW (Yongjun Wang), XZ, PW, YZ. Administrative support: XZ, YW (Yilong Wang), LL, PW, RJ, ZY. Provision of study materials or patients: all authors. Collection and assembly of data: AW. Data analysis and interpretation: AW. Manuscript writing: all authors. Final approval of manuscript: all authors.

Funding This work was supported by grants from the National Key R\&D Program of China (2016YFC0901002, 2017YFC1310901, 2018YFC1312903), grants from Beijing Municipal Science and Technology Commission (D171100003017002) and grants from the National Science and Technology Major Project (2017Z×09304018).

Competing interests None declared.

Patient consent for publication Not required.

Ethics approval Institutional Review Board (IRB) of Beijing Tiantan Hospital, Capital Medical University.

Provenance and peer review Not commissioned; externally peer reviewed.

Data availability statement All data relevant to the study are included in the article or uploaded as supplementary information. The data are available from XZ, Department of Neurology, Beijing Tiantan Hospital, Capital Medical University, Beijing, China. Tel: +86-10-59978350. Fax: +86-1059973383. Email address: zxq@vip.163.com. The data reuse is permitted under the corresponding author's permission.

Open access This is an open access article distributed in accordance with the Creative Commons Attribution Non Commercial (CC BY-NC 4.0) license, which permits others to distribute, remix, adapt, build upon this work non-commercially, and license their derivative works on different terms, provided the original work is properly cited, appropriate credit is given, any changes made indicated, and the use is non-commercial. See: http://creativecommons.org/licenses/by-nc/4.0/.

\section{ORCID iDs}

Yaqing Zhang http://orcid.org/0000-0003-4636-446X

Yongjun Wang http://orcid.org/0000-0002-9976-2341

Anxin Wang http://orcid.org/0000-0003-4351-2877

Liping Liu http://orcid.org/0000-0003-2943-055X

\section{REFERENCES}

1 Feigin VL, Lawes CMM, Bennett DA, et al. Worldwide stroke incidence and early case fatality reported in 56 population-based studies: a systematic review. Lancet Neurol 2009;8:355-69.
2 Keep RF, Hua Y, Xi G. Intracerebral haemorrhage: mechanisms of injury and therapeutic targets. Lancet Neurol 2012;11:720-31.

3 Krishnamurthi RV, Moran AE, Feigin VL, et al. Stroke prevalence, mortality and Disability-Adjusted life years in adults aged 20-64 years in 1990-2013: data from the global burden of disease 2013 study. Neuroepidemiology 2015;45:190-202.

4 Adeoye O, Broderick JP. Advances in the management of intracerebral hemorrhage. Nat Rev Neurol 2010;6:593-601.

5 Wang W-J, Lu J-J, Wang Y-J, et al. Clinical characteristics, management, and functional outcomes in Chinese patients within the first year after intracerebral hemorrhage: analysis from China national stroke Registry. CNS Neurosci Ther 2012;18:773-80.

6 van Asch CJ, Luitse MJ, Rinkel GJ, et al. Incidence, case fatality, and functional outcome of intracerebral haemorrhage over time, according to age, sex, and ethnic origin: a systematic review and meta-analysis. Lancet Neurol 2010;9:167-76.

7 Morotti A, Marini S, Lena UK, et al. Significance of admission hypoalbuminemia in acute intracerebral hemorrhage. J Neurol 2017;264:905-11.

8 Marini S, Morotti A, Lena UK, et al. Men experience higher risk of pneumonia and death after intracerebral hemorrhage. Neurocrit Care 2018;28:77-82.

9 Nzwalo H, Nogueira J, Félix AC, et al. Short-Term outcome of spontaneous intracerebral hemorrhage in Algarve, Portugal: retrospective hospital-based study. J Stroke Cerebrovasc Dis 2018;27:346-51.

10 Shah SV, Corado C, Bergman D, et al. Impact of poststroke medical complications on 30-day readmission rate. J Stroke Cerebrovasc Dis 2015;24:1969-77.

11 Johnston KC, Li JY, Lyden PD, et al. Medical and neurological complications of ischemic stroke: experience from the RANTTAS trial. RANTTAS Investigators. Stroke 1998;29:447-53.

12 Langhorne P, Stott DJ, Robertson L, et al. Medical complications after stroke: a multicenter study. Stroke 2000;31:1223-9.

13 Bae H-J, Yoon D-S, Lee J, et al. In-Hospital medical complications and long-term mortality after ischemic stroke. Stroke 2005;36:2441-5.

14 Heuschmann PU, Kolominsky-Rabas PL, Misselwitz B, et al. Predictors of in-hospital mortality and attributable risks of death after ischemic stroke: the German stroke registers Study Group. Arch Intern Med 2004;164:1761-8.

15 Saposnik G, Hill MD, O'Donnell M, et al. Variables associated with 7-day, 30-day, and 1-year fatality after ischemic stroke. Stroke 2008;39:2318-24.

16 Vernino S, Brown RD, Sejvar JJ, et al. Cause-Specific mortality after first cerebral infarction: a population-based study. Stroke 2003;34:1828-32.

17 Wang P-L, Zhao X-Q, Yang Z-H, et al. Effect of in-hospital medical complications on case fatality post-acute ischemic stroke: data from the China national stroke Registry. Chin Med J 2012;125:2449-54.

18 Wang P-L, Zhao X-Q, DU W-L, et al. In-Hospital medical complications associated with patient dependency after acute ischemic stroke: data from the China national stroke Registry. Chin Med J 2013;126:1236-41.

19 Wang P, Wang Y, Zhao X, et al. In-Hospital medical complications associated with stroke recurrence after initial ischemic stroke. Medicine 2016:95:e4929.

20 Wang Y, Cui L, Ji X, et al. The China national stroke Registry for patients with acute cerebrovascular events: design, rationale, and baseline patient characteristics. Int J Stroke 2011;6:355-61.

21 Brott T, Adams HP, Olinger CP, et al. Measurements of acute cerebral infarction: a clinical examination scale. Stroke 1989;20:864-70.

22 Lisk DR, Pasteur W, Rhoades H, et al. Early presentation of hemispheric intracerebral hemorrhage: prediction of outcome and guidelines for treatment allocation. Neurology 1994;44:133-9.

23 Lord AS, Lewis A, Czeisler B, et al. Majority of 30-day readmissions after intracerebral hemorrhage are related to infections. Stroke 2016:47:1768-71.

24 Morotti A, Marini S, Jessel MJ, et al. Lymphopenia, infectious complications, and outcome in spontaneous intracerebral hemorrhage. Neurocrit Care 2017;26:160-6.

25 Lindner A, Kofler M, Rass V, et al. Early predictors for infectious complications in patients with spontaneous intracerebral hemorrhage and their impact on outcome. Front Neurol 2019;10:817.

26 Qiu M, Sato S, Zheng D, et al. Admission heart rate predicts poor outcomes in acute intracerebral hemorrhage: the intensive blood pressure reduction in acute cerebral hemorrhage trial studies. Stroke 2016;47:1479-85. 